\title{
Schwannome de la langue : à propos d'un cas et revue de la littérature
}

\section{Schwannoma of the tongue: case report and literature review}

ANNE LACHERE, SIHAM KERRARY, ANDRE CHAINE, STEPHANIE BOUVIER, ARNAUD RIGOLET, CHLOE BERTOLUS

\section{RÉSUMÉ}

Les schwannomes ou neurilemmomes sont des tumeurs bénignes encapsulées, le plus souvent solitaires, issues des cellules de Schwann des gaines nerveuses. Dans la région cervico-faciale, $1 \%$ des schwannomes sont retrouvés dans la cavité buccale et surtout sur la langue.

Les auteurs rapportent le cas d'un schwannome localisé sur le bord latéral gauche de la langue. Une exérèse chirurgicale complète a été réalisée, et l'examen histopathologique de la pièce opératoire était en faveur d'un schwannome. Les suites ont été simples, et après 4 ans de recul, le patient ne présente pas de récidive.

Le diagnostic positif repose sur l'examen histopathologique. Le traitement consiste en une exérèse chirurgicale complète, les récidives sont exceptionnelles. (Med Buccale Chir Buccale 2009 ; 15: 195-198).

mots clés: langue, schwannome, neurilemmome, tumeurs nerveuses

médecine buccale chirurgie buccale VOL. $15, \mathrm{~N}^{\circ} 4$ 2009 page 195

\section{$\underline{\text { SUMMARY }}$}

Schwannoma or neurilemmoma is benign, slow, usually solitary and encapsulated tumor, originating from Schwann cells of the nerve sheath. Intraoral schwannoma accounts for $1 \%$ of head and neck area and are commonly seen at the tongue.

The authors report a case of schwannoma, located on the left lateral border of the tongue. Complete surgical excision of the tumor was performed and the histological evaluation of the excised specimen was consistent with schwannoma. The patient is under clinical control, with no signs of recurrence even after four years.

Diagnosis of the schwannoma is made by histological examination of the lesion. The treatment is surgical excision of the lesion. The recurrence after complete excision is rarely. (Med Buccale Chir Buccale 2009 ; 15: 195-198).

key words: tongue, schwannoma, neurilemmoma, neurogenic tumours 
Le schwannome, encore appelé neurinome, neurilemmome ou fibroblastome périneural, représente une tumeur bénigne issue des cellules de Schwann, d'origine neuro-ectodermique, se développant souvent au dépens du système nerveux périphérique moteur et sensitif.

$25 \%$ des schwannomes sont retrouvés dans la sphère cervico-faciale, mais seulement $1 \%$ dans la cavité orale ; la langue étant la structure la plus atteinte.

Les auteurs rapportent un cas de schwannome de la langue, discutent les différents diagnostics différentiels possibles, et précisent l'intérêt de l'examen histopathologique complété par un examen en immunohistochimie, qui seul permet de poser le diagnostic positif de schwannome bénin.

\section{CAS CLINIQUE}

médecine

buccale chirurgie buccale

VOL. $15, \mathrm{~N}^{\circ} 4$ 2009

page 196
II s'agissait d'un homme de 32 ans, sans antécédents pathologiques particuliers, qui s'est présenté en consultation dans le service de chirurgie maxillofaciale en mai 2005, pour le bilan d'une tuméfaction linguale évoluant depuis 6 mois. Le patient souffrait d'une simple gêne. L'examen retrouvait une tuméfaction de $4 \times 2 \mathrm{~cm}$, ferme et indolore, sur le bord latéral gauche de la langue. II n'existait pas de lésion de la muqueuse en regard (Fig. 1). Le bilan d'extension locorégional et général était négatif.

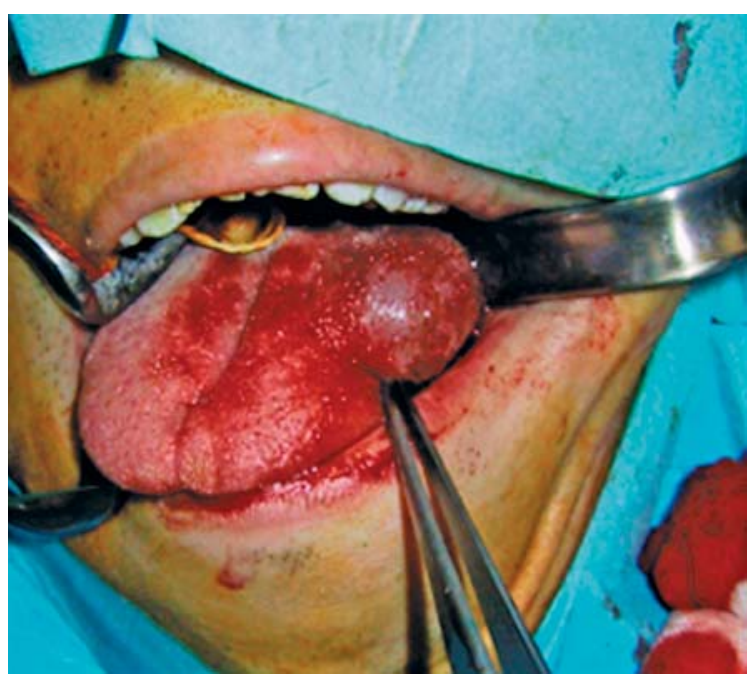

Figure 1 : Tumeur localisée sur le bord latéral gauche de la langue, recouverte par une muqueuse saine. Tumor located on the left lateral border of the tongue, covered with normal mucosa.
Le traitement a été chirurgical : exérèse complète de la tumeur encapsulée après avoir pratiqué une simple incision verticale en regard de la tumeur (Fig. 2 et 3).

L'examen histopathologique de la pièce opératoire qui mesurait $4 \times 2 \times 2 \mathrm{~cm}$, a permis de poser le diagnostic de schwannome bénin. Les suites postopératoires ont été simples. Après 4 ans de recul, on n'a observé aucune récidive clinique.

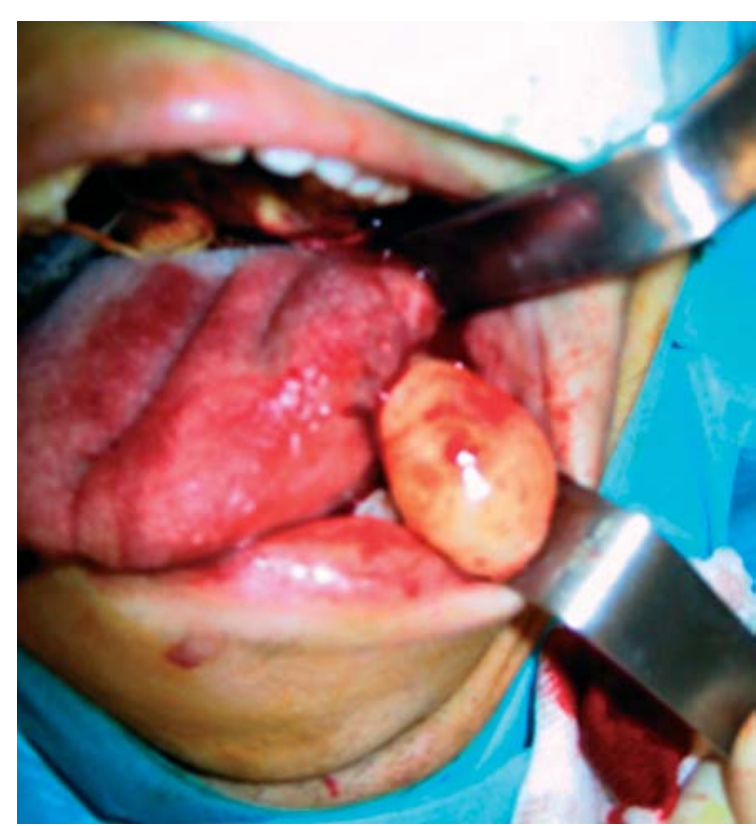

Figure 2 : Aspect encapsulé de la tumeur. Encapsulated tumor.

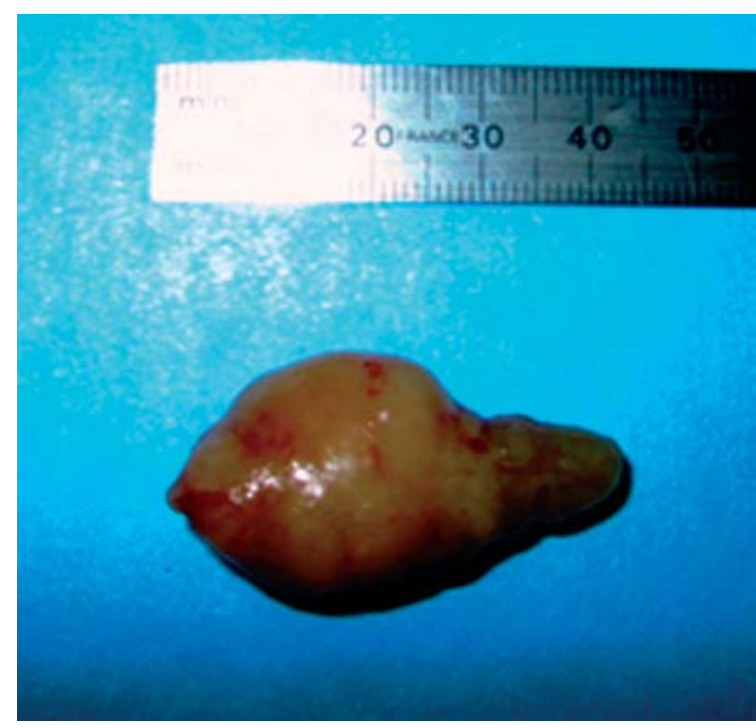

Figure 3 : Exérèse chirurgicale complète. Complete surgical excision. 


\section{DISCUSSION}

Les schwannomes sont des tumeurs issues des cellules nerveuses de Schwann, se développant surtout à partir des nerfs périphériques moteurs ou sensitifs. Ils peuvent survenir à tout âge, mais plus particulièrement entre 20 et 30 ans (67\% des cas) et il n'existe pas de prédominance de sexe ou de race ${ }^{[1]}$. 25\% des schwannomes se développent dans la région cervico-faciale ; seulement $1 \%$ d'entre eux intéressent la cavité buccale [2-5] ; ils représentent 1 à $9 \%$ de l'ensemble des tumeurs stomatologiques [2]. D'après la littérature, les schwannomes à localisation buccale intéressent la langue dans $50 \%$ des cas, surtout sa partie mobile, principalement les bords latéraux, alors que l'atteinte de la pointe est très rare. Dans les autres localisations on retrouve, par ordre décroissant, l'atteinte du palais, du plancher buccal, du vestibule, de la gencive, et de la lèvre $[2-4,6,7]$. L'atteinte osseuse est très rare. Fawcett et Dahlin rapportent 7 cas de schwannomes sur 3987 tumeurs osseuses [1].

D'étiologie inconnue, certains facteurs sont incriminés dans leur développement : les irritations chroniques, les traumatismes [8], et un traitement par radiothérapie ${ }^{[5,8]}$.

La lésion est généralement unique. Des schwannomes multiples (schwannomatose) peuvent s'observer dans la neurofibromatose de type II qui est caractérisée entre autres par un schwannome acoustique, bilatéral ou non [9].

Le schwannome bénin pose le problème diagnostique avec les autres tumeurs nerveuses, en particulier avec les névromes, constitués par une prolifération d'axones et de cellules de Schwann induite le plus souvent par un traumatisme local, comme par exemple, lors d'une intervention de Caldwell Luc. Mais aussi avec les tumeurs à cellules granuleuses d'Abrikossof, les gliomes, les neurothécomes, les méningiomes extracrâniens, les chondromes, les tumeurs neuroectodermiques mélanotiques [10]. En dehors des tumeurs nerveuses, le diagnostic différentiel se pose avec les lipomes, les léiomyomes, les hémangiomes, les lymphangiomes, et les tumeurs bénignes ou malignes des glandes salivaires.

Cliniquement, les schwannomes sont le plus souvent asymptomatiques, de croissance lente, mais ils peuvent être accompagnés de douleurs et/ou de paresthésies dans $50 \%$ des cas. D'autres symptômes ont été rapportés en fonction de la localisation tumorale, on peut noter l'apparition d'une dysphagie et d'une modification de la voix dans les schwannomes de la base de langue, des signes neurologiques comme une paralysie faciale lors de l'atteinte de rameaux du nerf facial, une baisse d'acuité auditive dans les neurinomes de l'acoustique, une dyspnée s'il y a une atteinte des nerfs IX, X, XI, ou une perte de la sensibilité linguale (atteinte du nerf lingual). Les nerfs les plus touchés sont les nerfs V, VII, VIII, IX, XI, XII. Pour la localisation linguale, l'examen montre une tuméfaction ferme et régulière, les ulcérations en regard sont exceptionnelles.

Radiologiquement, les images obtenues sont peu spécifiques. L'échographie enregistre un signal homogène, hypoéchogène avec renforcement postérieur. Le scanner montre une masse hétérogène ou homogène, isodense par rapport aux parties molles, avec prise de contraste hétérogène $[4,6]$. L'IRM montre une lésion isointense aux muscles en T1 et hyperintense en T2, avec prise de contraste après injection de gadolinium [11]. L'imagerie permet d'appréhender l'extension de la tumeur.

Le traitement est chirurgical, il consiste en une exérèse complète de la tumeur sans marge de sécurité, si elle est bien encapsulée.

L'examen histopathologique de la pièce montre une tumeur entourée d'une capsule fibreuse, constituée de deux populations cellulaires, les cellules Antoni A (majoritaires) et les cellules Antoni $B[4,7]$. Les cellules $A$, fusiformes, enchâssées dans un tissu conjonctif riche en collagène, sont orientées parallèlement, groupées en faisceaux compacts autour d'une zone centrale acellulaire. Cet ensemble forme les corpuscules de Verocay. Les noyaux de ces cellules sont alignés en palissade. Les cellules $B$ sont moins bien ordonnées, réticulées, au sein d'un tissu conjonctif lâche et de fibres entourant des microkystes. La tumeur est peu vascularisée, les vaisseaux dilatés, avec des parois épaissies et hyalinisées. L'examen histopathologique permet d'écarter le diagnostic de schwannome malin [12, 13], dont la fréquence est de l'ordre de 5 à $10 \%$ de l'ensemble des sarcomes des tissus mous, en montrant des caractères évocateurs médecine buccale chirurgie buccale VOL. $15, N^{\circ} 4$ 2009 page 197 
médecine

buccale chirurgie buccale

VOL. $15, \mathrm{~N}^{\circ} 4$ 2009

page 198 mais moins spécifiques : présence de bandes et de nodules hyalins autour desquels les cellules peuvent se disposer en rosettes, extension péri et intra-neurale de la tumeur, prolifération cellulaire autour des vaisseaux faisant hernie dans la lumière vasculaire, éléments hétérotopiques (environ $15 \%$ des cas), le plus souvent sous forme d' îlots de cartilage ou d'os mature, plus rarement sous forme de fibres musculaires striées (tumeur de Triton) ou de glandes mucosécrétantes (schwannome malin glandulaire). L'aspect des cellules est un argument important pour le diagnostic. Les cellules fusiformes ont des contours irréguliers avec des noyaux asymétriques courbés, ondulés ou en virgule. Le cytoplasme est peu coloré, à limites mal visibles. L'étude immunohistochimique, quelque soit la nature du schwannome, retrouve une réaction positive pour la protéine S100 [14].

Le schwannome ancien est une variante rare du schwannome bénin caractérisé par des remaniements dégénératifs histologiques : calcifications, hyalinisation, formations kystiques. II existe aussi des zones d'atypie cellulaire faisant porter à tort le diagnostic de malignité [15].

Il existe un risque de transformation maligne des schwannomes bénins, qui varie entre 8 et $13,9 \%$ car, en augmentant de taille, la vascularisation devient insuffisante, favorisant la transformation maligne [15]. Malgré ce risque de transformation maligne, les schwannomes bénins gardent un bon pronostic si l'exérèse chirurgicale est complète, les récidives sont exceptionnelles. Par contre, le schwannome malin est une tumeur de mauvais pronostic avec une survie globale de 20 à $25 \%$ en cas de maladie de Recklinghausen et de $50 \%$ en cas de tumeur isolée. Le mauvais pronostic dans la maladie de Recklinghausen est lié au siège des tumeurs (plus souvent au niveau du tronc ou proximales), à la taille et au grade plus élevé, et au fait que certains patients développent plusieurs sarcomes simultanément. Les récidives locales sont fréquentes, et les métastases (poumons, foie, peau et os) apparaissent habituellement dans les deux ans après le diagnostic [12, 13] .

\section{RÉFÉRENCES}

1 - Buric N, Jovanovic G, Pesic Z, Krasic D, Radovanovic Z, Mihailovic D, Tijanic M. Mandible schwannoma (neurilemmoma) presenting as periapical lesion. Dentomaxillofac Radiol 2009 ; 38 : 178-81.

2 - Pfeifle R, Baur DA, Paulino A, Helman J. Schwannoma of the tongue: report of 2 cases. J Oral Maxillofac Surg $2001 ; 59: 802-4$.

3 - Chuang HC, Su CY, Lui CC, Hwang CF, Chien CY. Lingual nerve neurilemmoma. J Otolaryngol 2004 ; 33 : 132-4.

4 - Hsu YC, Hwang CF, Hsu RF, Kuo FY, Chien CY, Schwannoma (neurilemmoma) of the tongue. Acta OtoLaryngol 2006 ; 126 : 861-5.

5 - Cinar F, Cinar S, Harman G, Schwannoma of the tip of the tongue in a child. Plast Reconstruct Surg 2004 ; $114: 1657-8$

6 - Arda HN, Akdogan O, Arda N, Sarikaya Y. An unusual site for an intraoral schwannoma: a case report. Am J Otolaryngol $2003 ; 24$ : 348-50.

7 - Ying YLM, Zimmer LA, Myers EN. Base of tongue schwannoma: a case report. Laryngoscope $2006 ; 116$ : 1284-7.

8 - Hwang K, Kim SG, Ahn SI, Lee SI. Neurilemmoma of the tongue. J Craniofac Surg 2005 ; 16 : 859-61.

9 - Avcu N, Kansu O, Uysal S, Kansu H. Cranio-orbitaltemporal neurofibromatosis with cerebral hemiatrophy

presenting as an intraoral mass: a case report. J Calif Dent Assoc 2009 ; 37 : 119-21.

10 - Zachariades N, Mezitis M, Vairaktaris E, Triantafyllou D, Skoura-Kafoussia C, Konsolaki-Agouridaki E, Hadjiolou E, Papavassiliou D. Benign neurogenic tumors of the oral cavity. Int J Oral Maxillofac Surg 1987 ; 16 : 70-6.

11 - Cai W, Kassarjian A, Bredella MA, Harris GJ, Yoshida H, Mautner VF, Wenzel R, Plotkin SR. Tumor burden in patients with neurofibromatosis types 1 and 2 and schwannomatosis: determination on whole-body MR images. Radiology 2009 ; 250 : 665-73.

12 - Guglielmotti M B, Pena C, Dominguez FV. Malignant schwannoma of the gingival. Int J Oral Maxillofac Surg $1987 ; 16: 492-5$.

13 - Scheithauer BW, Woodruff JM, Erlandson RA. Tumors of the peripheral nervous system. Atlas of tumor pathology, AFIP, 3rd series, 1999

14 - Ferreti Bonan PR, Martelli H Jr, Nogueira Dos Santos LA, Comini Mol V, Paes De Almeida O. Multinodular neurilemmoma of the tongue: a case report with differential immunohistochemical profile. Minerva Stomatol 2008 ; $57: 71-5$.

15 - Zachariades N, Skoura C, Papageorgiou G, Chrissomali E. Giant ancient neurilemmoma of cervical region: report of case. J Oral Maxillofac Surg 2001 ; 59 : 668-72. 